\title{
Guide-wire replacement of a mini-midline catheter with a central venous catheter: A retrospective study on $\mathbf{6 3}$ cases
}

The Journal of Vascular Access

$1-4$

(C) The Author(s) 2020

Article reuse guidelines:

sagepub.com/journals-permissions DOI: I0.1 I77/II29729820944066 journals.sagepub.com/home/jva

(S)AGE

\author{
Lucio Brugioni' (1D, Elisabetta Bertellini², Mirco Ravazzini², \\ Marco Barchetti ${ }^{2}$, Andrea Borsatti ${ }^{2}$, Pietro Martella ${ }^{2}$, \\ Massimo Girardis ${ }^{2}$, Francesco Serra ${ }^{2}$, Angelo Tricoli ${ }^{2}$, \\ Matteo Nicolini ${ }^{2}$, Marcello Bianchini ${ }^{2}$, Filippo Schepis ${ }^{2}$, \\ Giovanni Tazzioli', Giovanni Pinelli ${ }^{2}$, Elisa Romagnoli' \\ and Roberta Gelmini
}

\begin{abstract}
Background: Achieving a reliable venous access in a particular subset of patients and/or in emergency settings can be challenging and time-consuming. Furthermore, many hospitalized patients do not meet the criteria for central venous catheter positioning, unless an upgrade of the treatment is further needed. The mini-midline catheter has already showed to be reliable and safe as a stand-alone device, since it is easily and rapidly inserted and can indwell up to I month.

Methods: In this further case series, we retrospectively evaluated data from 63 patients where a previously inserted mini-midline catheter was upgraded to a central venous catheter (the devices inserted in the arm replaced by peripherally inserted central catheter and others inserted "off-label" in the internal jugular replaced by single lumen centrally inserted central catheter), being used as introducer for the Seldinger guidewire.

Results: The guidewire replacement was been made even early (after I day) or late (more than I0 days), usually following a need for an upgrade in treatment. No early or late complications were reported.

Conclusion: According to the preliminary data we collected, this converting procedure seems to be feasible and riskfree, since neither infectious nor thrombotic complications were reported.
\end{abstract}

\section{Keywords}

JLB, mini-midline, peripherally inserted central catheter, centrally inserted central catheter, case series, ultrasoundguided

Date received: 25 April 2020; accepted: 24 June 2020

\section{Background}

The lack of venous asset in patients who are frequently hospitalized, obese, or underweight is a common problem in ordinary wards as well as in intensive care units (ICUs). ${ }^{1-3}$ This clinical feature is called difficult venous access (DIVA), defined as a clinical condition in which several attempts are expected or required to obtain and maintain peripheral venous access. ${ }^{4}$ The ideal solution in these patients is often the positioning of a peripheral venous access device via ultrasound guidance (such as a mini-midline).

In addition, it is known that a considerable proportion of patients will require a treatment upgrade (even for limited periods). According to the principles of proper planning of vascular access, ${ }^{5}$ this could often imply the

\footnotetext{
'Medicina d'Emergenza-Urgenza, Università degli Studi di Modena e Reggio Emilia, Modena, Italy

${ }^{2}$ Facoltà di Medicina e Chirurgia, Università degli Studi di Modena e Reggio Emilia, Modena, Italy

Corresponding authors:

Lucio Brugioni, Medicina d'Emergenza-Urgenza, Università degli Studi di Modena e Reggio Emilia, 4I I2I Modena, Italy.

Email: brugioni.lucio@aou.mo.it

Elisa Romagnoli, Medicina d'Emergenza-Urgenza, Università degli Studi di Modena e Reggio Emilia, 4I I 2 I Modena, Italy.

Email: elyrom80@gmail.com
} 
need for a switch from peripheral venous devices (minimidline and midline) to central venous catheters type CICC (centrally inserted central catheter) or PICC (peripherally inserted central catheter).

The CICC and PICC insertion procedures are well codified and require trained personnel; ${ }^{6}$ in particular, in the last decade, there has been a progressive increase in PICCs, ${ }^{7}$ which can be used for all the CICC indications (parenteral nutrition, chemotherapy, and long-term antibiotic therapy) but with less invasiveness. ${ }^{8-12}$ However, the PICC and CICC insertion is associated with logistical difficulties and the likely occurrence of complications. ${ }^{13-16}$

We started using the mini-midline catheters in 2015 following the experience of Teismann et al. ${ }^{17}$ who in 2013 published about the "easy IJ" using standard peripheral catheters to achieve internal jugular vein (IJV) access.

In our institution, mini-midline catheters ${ }^{18}$ are commonly inserted both in the ordinary wards and in the ICUs and sub-ICUs. The placement of these devices (mini-midlines "off-label" in the IJV or in the vein of the arms) is especially indicated in patients with DIVA and in those who are expected to need venous access for prolonged periods.

One possible advantage of this device lies in the possibility of using it for the positioning of CICC and PICC without resorting to new venipuncture but replacing on a guidewire (Seldinger technique) this peripheral route with a central route. In this retrospective study, we evaluated the feasibility and safety of this maneuver.

\section{Methods}

From June 2015 and March 2018, we collected retrospectively data from all patients in whom the placement of CICC and PICC had occurred by replacement on a guidewire through a previously inserted mini-midline $\left(\mathrm{JLB}^{\circledR}\right.$ catheter; DeltaMed, Inc., Somerset, NJ, USA, only type of mini-midline used on the study). The patients were afferent to three different operative units: (a) intensive internal medicine of the Policlinico Hospital in Modena, (b) ICU of the new Sant'Agostino Estense Civil Hospital in Modena, and (c) emergency department of the new Sant'Agostino Estense Civil Hospital in Modena.

The initial insertion of the mini-midline device, by ultrasound-guided venipuncture of the IJV or basilic vein, was carried out by physicians and nurses specifically trained in this maneuver.

The mini-midline positioning technique involves use aseptic technique; after ultrasound exploration of the neck or the arm, we applied chlorhexidine $2 \%$ alcoholic solution, sterile gloves, and sterile ultrasound cover probe of a similar device. To position mini-midline, we used direct technique venipuncture (cannulation of the IJV in the neck - with an out-of-plane or in-plane technique - or the basilic vein to the arm-always with out-of-plane technique) as for standard peripheral cannula. The cannulation of the vein is confirmed by direct blood aspiration and direct ultrasound visualization of the catheter within the vein. The catheter is then fixed to the skin by sterile sutureless devices and/or transparent semipermeable membranes. At the end of the procedure, a further ultrasound check is performed to rule out early local complications (e.g. pneumothorax after jugular puncture or local hematomas).

The decision to initially place a mini-midline was dictated by the need to place venous access in emergency setting, as it was impossible to find other accesses and there were no criteria for CICC or PICC positioning at that time.

The placement of a central venous catheter by minimidline catheter-guided replacement was performed by doctors or nurses at the relative operating units. The reasons were always established by the occurrence of changes in therapy that required an upgrade to a central venous access device.

Initially, the guide replacement maneuver involved an ultrasound check of the vessel using a high-frequency linear probe, to rule out the presence of in situ venous thrombosis. Subsequently, for the over-the-wire procedure, a full sterile technique using sterile field, glows gloves, cap, mask as for usual central venous catheterization was necessary. Using chlorhexidine $2 \%$ alcoholic solution, we cleaned and disinfected the exit site and the device before removing the anti-reflux cap a full sterile technique, sterile field, gloves, cap, and mask was necessary as for usual central venous catheterization. We cleaned and disinfected the exit site and the device before removing the anti-reflux cap, using chlorhexidine $2 \%$ alcoholic solution. The maneuver, therefore, included the insertion of the guidewire in the mini-midline catheter, typically using $0.035^{\prime \prime} \mathrm{J}$ guides in the case of CICCs and straight guides but with a "floppy straight tip" of 0.018 " in the case of PICCs; once the mini-midline catheter is removed, the guidewire was used to position the new device. Tip confirmation in all cases of this study was confirmed using chest X-ray as for CICC and PICC.

All the PICCs and CICCs were fixed by sutureless devices (no catheter was fixed with stitches) and the site covered with a transparent semipermeable membrane. We used multiple brands 7Fr 20-cm polyurethane multi-lumen central vascular catheter and $4 \mathrm{Fr}$ single-lumen polyurethane PICCs.

The primary outcomes were feasibility and safety of central venous catheter placement by mini-midline catheter-guided replacement; secondary outcome was late thrombotic or infectious complications. The ethical committee approval was obtained.

\section{Results}

We collected retrospectively data from 63 patients in whom the central venous catheter was placed by replacement through a mini-midline catheter: 36 CICCs and 27 PICCs were positioned (Table 1). 
The positioned mini-midline catheters had a caliber of $16 \mathrm{G}(\mathrm{n}=16), 17 \mathrm{G}(\mathrm{n}=22)$, or $18 \mathrm{G}(\mathrm{n}=25)$ and a length of $6 \mathrm{~cm}(\mathrm{n}=4), 7 \mathrm{~cm}(\mathrm{n}=52)$, or $8 \mathrm{~cm}(\mathrm{n}=7)$ (Table 2).

All of the positioned CICCs were 7Fr multi-lumen catheters given the acute situation, while the positioned PICCs were 4Fr. The indwelling time of the mini-midline catheter in the IJV before the CICC replacement was between 24 and $192 \mathrm{~h}$ with an average of $93.3 \mathrm{~h}$ and a median of $96 \mathrm{~h}$. The replacement of the mini-midline catheter with a PICC was instead carried out after a time between 48 and $240 \mathrm{~h}$ with an average of $143.1 \mathrm{~h}$ and a median of $144 \mathrm{~h}$.

No complications were reported, neither during the insertion of CICC nor during the insertion of PICC. The CICCs remained in situ for a time between 7 and 16 days (average $242 \mathrm{~h}$, median $240 \mathrm{~h}$ ).

The PICCs instead remained in situ for 30-115 days (average 70.96 days, median 69 days). No late complications occurred in either group; in particular, there were no cases of infection or thrombosis. The maneuver was performed in patients of both sexes (female: $\mathrm{n}=34$ ); the average age was 75.7 (73.2 years in the CICC group and 79 years in the PICC group).

\section{Discussion}

In patients who lack venous asset and need to place venous access in urgency/emergency setting, if there is no need for central access, the choices available are very limited. The most frequent option is the positioning of central venous

Table I. Sex and mean age of the patient.

\begin{tabular}{lllll}
\hline $\begin{array}{l}\text { Type of device } \\
\text { replace }\end{array}$ & Male (n) & $\begin{array}{l}\text { Mean } \\
\text { age }\end{array}$ & Women (n) & $\begin{array}{l}\text { Mean } \\
\text { age (aa) }\end{array}$ \\
\hline CICC & 17 & 72.9 & 19 & 73.5 \\
PICC & 12 & 76.6 & 15 & 81.1 \\
Total & 29 & 74.4 & 34 & 76.9 \\
\hline
\end{tabular}

CICC: centrally inserted central catheter; PICC: peripherally inserted central catheter. access with central insertion (CICC). However, this choice brings several problems: (a) often, a central device is not strictly indicated and could be configured as an "overtreatment" whose invasiveness is not justified by clinical needs; (b) trained personnel specifically trained in CICC placement may be missing; (c) emergency settings may not be compatible with the time required for the placement of a CICC.

Moreover, the placement of a PICC - even if less invasive than a CICC - is never advisable in urgency, in addition to having even longer implementation times.

This study, despite all limitations of small sample size and retrospectivity and "off-label" use of mini-midline catheters with internal jugular incannulation, offers a solution that satisfies the condition of emergency venous access positioning, through a safe and relatively simple maneuver. At the same time, mini-midline catheters have the advantage of great safety and reliability even for the subsequent positioning of a central venous catheter by guide replacement.

The placement of a CICC or a PICC by mini-midline catheter-guided replacement is subjected to some important precautions:

- A guided replacement will be contraindicated in the presence of a suspected or diagnosed infection associated with the mini-midline catheter. For example, the presence of a grade 2 inflammation according to the Visual Exit Score ${ }^{19}$ is a contraindication.

- An ultrasound examination must be performed to rule out a thrombosis associated with the mini-midline catheter, since this also involves a contraindication to the switch to central venous catheter.

- As recommended by the Centers for Disease Control and Prevention (CDC) guidelines, ${ }^{20}$ the guidewire replacement requires, immediately after the insertion of the guidewire and the removal of the mini-midline catheter, a new skin disinfection with $2 \%$ chlorhexidine around the guide, the change of the operator's sterile gloves, and the resuming of the maneuver with maximum barrier precautions.

Table 2. Insertion site, insertion time, and mini-midline device features.

\begin{tabular}{|c|c|c|c|c|}
\hline $\begin{array}{l}\text { The first type of } \\
\text { device positioned }\end{array}$ & $\begin{array}{l}\text { Side of insertion } \\
\text { (n) }\end{array}$ & $\begin{array}{l}\text { Mean time of } \\
\text { insertion (s) }\end{array}$ & $\begin{array}{l}\text { Diameter } \\
\text { (n) }\end{array}$ & $\begin{array}{l}\text { Length of the } \\
\text { catheter }(n)\end{array}$ \\
\hline \multirow[t]{8}{*}{$\mathrm{JLB}^{\circledR}$} & \multirow{3}{*}{$\begin{array}{l}\text { Right internal } \\
\text { jugular vein }(36)\end{array}$} & \multirow[t]{3}{*}{330} & \multirow[t]{3}{*}{$5 F r(16)$} & $6 \mathrm{~cm}(2)$ \\
\hline & & & & $7 \mathrm{~cm}(12)$ \\
\hline & & & & $8 \mathrm{~cm}(2)$ \\
\hline & & & \multirow[t]{3}{*}{ 4.4Fr (22) } & $6 \mathrm{~cm} \mathrm{(2)}$ \\
\hline & \multirow[t]{4}{*}{ Basilic vein (27) } & \multirow{4}{*}{361.2} & & $7 \mathrm{~cm}(\mathrm{I} 7)$ \\
\hline & & & & $8 \mathrm{~cm} \mathrm{(3)}$ \\
\hline & & & \multirow[t]{2}{*}{$3.8 \mathrm{Fr}(25)$} & $7 \mathrm{~cm}(23)$ \\
\hline & & & & $8 \mathrm{~cm} \mathrm{(2)}$ \\
\hline
\end{tabular}


If these precautions are adopted, as demonstrated by our experience, the risk of thrombotic or infectious complications for CICC and PICC positioned with this technique is almost nil.

\section{Conclusion}

Our experience suggests that the transition from mini-midline catheters to CICC and to PICC is a feasible, simple, and safe procedure, even in relatively elderly patients.

It is, therefore, possible to convert peripheral venous access into central venous access without the need for new venipuncture, with a large margin of safety: the absence of infections affecting the PICCs and CICCs positioned in this way is an indicator of maneuver's safety.

\section{Declaration of conflicting interests}

The author(s) declared no potential conflicts of interest with respect to the research, authorship, and/or publication of this article.

\section{Funding}

The author(s) received no financial support for the research, authorship, and/or publication of this article.

\section{ORCID iD}

Lucio Brugioni (iD https://orcid.org/0000-0002-0217-8545

\section{References}

1. Halvorson EE, Case D, Skelton JA, et al. Vascular access in critically III pediatric patients with obesity. Pediatric Critic Care Med 2018; 19(1): 1-8.

2. Sebbane M, Claret PG, Lefebvre S, et al. Predicting peripheral venous access difficulty in the emergency department using body mass index and critical evaluation of venous accessibility. J Emerg Med 2013; 44(2): 299-305.

3. Brandt HGS, Jepsen CH, Hendriksen OM, et al. The use of ultrasound to identify veins for peripheral venous access in morbidly obese patients. Dan Med J 2016; 63(2): A5191.

4. Rauch DD, Eldridge D, Mace S, Schears G, et al. Periferal difficult venous access in children. Clin Pediatr 2009; 48(9): 895-901.

5. Barton AJ, Danek G, Johns P, et al. Improving patient outcomes through CQI: vascular access planning. J Nurs Care Qual 1998; 13(2): 77-85.

6. Krein SL, Kuhn L, Ratz D, et al. Use of designated nurse PICC teams and CLABSI prevention practices among U.S. hospitals: a survey-based study. J Patient Safe 2019; 15: 293-295.

7. Zingg W, Sandoz L, Inan C, et al. Hospital-wide survey of the use of central venous catheters. J Hosp Infect 2011; 77(4): 304-308.

8. Chakravarthy SB, Rettmann J, Markewitz BA, et al. Peripherally inserted central catheter (PICC)-associated upper-extremity deep venous thrombosis (UEDVT) in critical-care setting. Chest 2005; 128(4 Suppl. S): 193S-194S.

9. Cowl CT, Weinstock JV, Al-Jurf A, et al. Complications and cost associated with parenteral nutrition delivered to hospitalized patients through either subclavian or peripherally inserted central catheters. Clin Nutr 2000; 19(4): $237-$ 243.

10. Safdar N and Maki DG. Risk of catheter-related bloodstream infection with peripherally inserted central venous catheters used in hospitalized patients. Chest 2005; 128(2): 489-495.

11. Bottino J, McCredie KB, Groschel DH, et al. Long-term intravenous therapy with peripherally inserted silicone elastomer central venous catheters in patients with malignant diseases. Cancer 1979; 43(5): 1937-1943.

12. Giuffrida DJ, Bryan-Brown CW, Lumb PD, et al. Central vs peripheral venous catheters in critically ill patients. Chest 1986; 90(6): 806-809.

13. Graham DR, Keldermans MM, Klemm LW, et al. Infectious complications among patients receiving home intravenous therapy with peripheral, central, or peripherally placed central venous catheters. Am J Med 1991; 91(3B): 95S-100S.

14. Monreal M, Lafoz E, Ruiz J, et al. Upper-extremity deep venous thrombosis and pulmonary embolism: a prospective study. Chest 1991; 99(2): 280-283.

15. Evans RS, Sharp JH, Linford LH, et al. Reduction of peripherally inserted central catheter-associated DVT. Chest 143; 627-633.

16. Pikwer A, Akeson J and Lindgren S. Complications associated with peripheral or central routes for central venous cannulation. Anaesthesia 2012; 67(1): 65-71.

17. Teismann NA, Knight RS, Rehrer M, et al. The ultrasoundguided "peripheral IJ": internal jugular vein catheterization using a standard intravenous catheter. J Emerg Med 2013; 44(1): 150-154.

18. Brugioni L, Barchetti M, Tazzioli G, et al. A new device for ultrasound-guided peripheral venous access. J Vasc Access 2019; 20: 325-328.

19. Gorski LA. The 2016 infusion therapy standards of practice. Home Healthc Now 2017; 35(1): 10-18.

20. O'Grady NP, Alexander M, Burns LA, et al. Guidelines for the prevention of intravascular catheter-related infections, 2011: Healthcare Infection Control Practices Advisory Committee. Am J Infect Control 2011; 39(4 Suppl. 1): S1-S34. 